\title{
Kaempferol as a flavonoid induces osteoblastic differentiation via estrogen receptor signaling
}

\author{
Ava Jiangyang Guo', Roy Chiyan Choi', Ken Yuzhong Zheng', Vicky Ping Chen', Tina Tingxia Dong ${ }^{1}$, \\ Zheng-Tao Wang ${ }^{2}$, Günter Vollmer ${ }^{3}$, David Taiwai Lau' ${ }^{1}$ and Karl Wah-keung Tsim*
}

\begin{abstract}
Background: Flavonoids, a group of compounds mainly derived from vegetables and herbal medicines, chemically resemble estrogen and some have been used as estrogen substitutes. Kaempferol, a flavonol derived from the rhizome of Kaempferia galanga L., is a well-known phytoestrogen possessing osteogenic effects that is also found in a large number of plant foods.

The herb K. galanga is a popular traditional aromatic medicinal plant that is widely used as food spice and in medicinal industries. In the present study, both the estrogenic and osteogenic properties of kaempferol are evaluated.

Methods: Kaempferol was first evaluated for its estrogenic properties, including its effects on estrogen receptors. The osteogenic properties of kaempferol were further determined its induction effects on specific osteogenic enzymes and genes as well as the mineralization process in cultured rat osteoblasts.

Results: Kaempferol activated the transcriptional activity of pERE-Luc (3.98 \pm 0.31 folds at $50 \mu \mathrm{M})$ and induced estrogen receptor $\alpha(E R \alpha)$ phosphorylation in cultured rat osteoblasts, and this ER activation was correlated with induction and associated with osteoblast differentiation biomarkers, including alkaline phosphatase activity and transcription of osteoblastic genes, e.g., type I collagen, osteonectin, osteocalcin, Runx2 and osterix. Kaempferol also promoted the mineralization process of osteoblasts $(4.02 \pm 0.41$ folds at $50 \mu \mathrm{M})$. ER mediation of the kaempferolinduced effects was confirmed by pretreatment of the osteoblasts with an ER antagonist, ICI 182,780, which fully blocked the induction effect.
\end{abstract}

Conclusion: Our results showed that kaempferol stimulates osteogenic differentiation of cultured osteoblasts by acting through the estrogen receptor signaling.

\section{Background}

Estrogen is known to play a significant role in bone metabolism in addition to its central roe in the reproductive system [1]. The osteoprotective effects of estrogen have been attributed mainly to its inhibitory action resorption of bone and stimulation of bone formation $[2,3]$. The drastic decrease in estrogen that accompanies menopause with the elevation of bone resoption caused by a rise in osteoclastogenesis is the most common cause of osteoporosis in women [4]. Clinically, estrogen replacement therapy has long been considered as the

\footnotetext{
* Correspondence: botsim@ust.hk

'Division of Life Science and Center for Chinese Medicine, State Key Laboratory of Molecular Neuroscience, The Hong Kong University of Science and Technology, Clearwater Bay, New Territories, Hong Kong SAR, China Full list of author information is available at the end of the article
}

first-line therapy for preventing and treating osteoporosis in post-menopausal women. However, estrogen treatment is linked with an increased risk of breast and uterine cancer [4].

Flavonoids, a group of naturally occurring plant secondary metabolites that are commonly found in fruits, vegetables and Chinese herbs, have been shown to exert a protective effect against post-menopausal bone loss [5-7]. The flavonol kaempferol, which is derived from the rhizome of Kaempferia galanga L., has been reported to possess various biological activities. The herb $K$. galanga is a popular traditional aromatic medicinal plant used in Asian countries, including China and Japan [8]. Traditionally, kaempferol is used to treat hypertension, abdominal pains, headache, and rheumatism. Kaempferol is determined to inhibit osteoclastic

\section{Biomed Central}


bone re-sorption in vitro [9] and to promote differentiation and mineralization of osteoblast-like cells $[10,11]$. However, the precise mechanism of action of kaempferol on bone homeostasis is not clearly known.

This study aims to investigate the estrogenic and osteogenic effects of kaempferol in primary cultured osteoblasts and evaluate whether the compound has estrogenic effect, particularly osteogenesis by inducing the enzymatic activity of alkaline phosphatase (ALP), which is an indicative osteoblast differentiation marker, and transcription of osteogenesis-associated genes, including type I collagen (COL1A1), osteonectin and osteocalcin, and two essential transcription factors (Runx2 and osterix) in cultured cells [12]. This study will determine whether effect of kaempferol on the mineralization process of osteoblasts is mediated by estrogen receptors (ERs) and not by a $\mathrm{Wnt} / \beta$-catenin signaling pathway.

\section{Materials and methods Chemicals}

Kaempferol was purchased from Wakojunyaku (Osaka, Japan) and had a purity of over $98 \%$. It was dissolved in dimethyl sulfoxide (DMSO) to give a stock solution of $100 \mathrm{mM}$. 17 3 -estradiol, ICI 182,780 and p-nitrophenylphosphate (pNPP) were purchased from Sigma (St. Louis, MO, USA). Recombinant human Dickkopf related protein 1 (DKK-1) and recombinant human Wnt-3a were purchased from Tocris Bioscience (Ellisville, MO, USA).

\section{Cell culture}

Rat primary osteoblasts were cultured and prepared by a previously described method [13] with minor modifications [14]. In brief, postnatal day 1 rats were decapitated to collect calvariae. Tissues were sequential digested by $1 \%$ trypsin for 10 minutes, $0.2 \%$ collagenase for $20 \mathrm{~min}$ utes and another freshly prepared $0.2 \%$ collagenase for 45 minutes. The supernatant was collected after centrifugation for 5 minutes at $1500 \mathrm{rpm}(200 \times g)$. Osteoblastic cells were re-suspended and maintained in modified Eagle's medium $\alpha$ (MEM $\alpha)$, supplemented with $10 \%$ fetal bovine serum, $2 \mathrm{mM}$ L-glutamine, 100 $\mathrm{U} / \mathrm{mL}$ penicillin and $100 \mu \mathrm{g} / \mathrm{mL}$ streptomycin in a humidified $\mathrm{CO}_{2}(5 \%)$ incubator at $37^{\circ} \mathrm{C}$. Before the cells were plated, they were washed with phosphate-buffered saline, and the medium was changed to MEM $\alpha$ (phenol red free) containing 5\% charcoal dextran-treated fetal bovine serum for 2 days. The rat primary osteoblasts were then seeded and treated with different drugs at various concentrations for predetermined time periods. In pre-treatment with an ER antagonist (ICI 182,780 or DKK-1), the antagonist was first added to the cultured osteoblasts for 1 hour, and then the tested drug was applied without washing out the antagonists. Reagents for cell cultures were purchased from Invitrogen Technologies (Carlsbad, CA, USA).

\section{Estrogenic activity and ER phosphorylation assays}

Three repeats of estrogen responsive elements (ERE: 5'GGT CAC AGT GAC ' C-3') were synthesized as described previously $[15,16]$ and then subcloned into a promoter-reporter vector PTAL-Luc (Clontech, Mountain View, CA, USA) which has a downstream firefly luciferase gene; this DNA construct was named pERELuc. Transient transfection of osteoblasts with the cDNA constructs was performed with Lipofectamine Plus reagent (Invitrogen, CA, USA), according to the manufacturer's instructions. Activation of luciferase gene expression driven by pERE-Luc was performed by a commercial kit (Tropix Inc., Bedford, MA, USA) [16]. The luminescent reaction was quantified in a Tropix TR717 ${ }^{\mathrm{TM}}$ microplate luminometer [Applied Biosystems, Bedford, MA], and the activity was expressed as absorbance (up to $560 \mathrm{~nm}$ ) per milligram of protein. This luciferase assay was also applied to a Wnt-responsive element, pWRE-Luc (five repeats of GAT CAA A) that was used here to test Wnt-induced signaling [12]. The phosphorylation of ER $\alpha$ (at serine 118) was determined by Western blot. Cultures of primary osteoblasts were serum starved for 3 hours prior to the addition of kaempferol. After treatment, the cultures were collected immediately in lysis buffer containing $125 \mathrm{mM}$ Tris- $\mathrm{HCl}$ (pH 6.8), 2\% sodium dodecyl sulfate (SDS), 10\% glycerol and $200 \mathrm{mM}$ 2-mercaptoethanol, and the proteins were subjected to SDS-PAGE analysis. After transfer, the membrane was incubated with anti-phospho-ER $\alpha$-S118 antibody (1:2000; Upstate, Lake Placid, NY, USA) and anti-total $\mathrm{ER} \alpha$ antibody (1:1000; Upstate) at $4{ }^{\circ} \mathrm{C}$ for 12 hours for protein detection. The immuno-complexes were visualized and quantified by the enhanced chemiluminescence method (GE Healthcare) as described previously $[12,15]$.

\section{ALP and mineralization assays}

Treated osteoblasts were placed in lysis buffer containing $0.2 \%$ Triton $\mathrm{X}-100,1 \mathrm{mM}$ dithiothreitol and 100 $\mathrm{mM}$ potassium phosphate buffer ( $\mathrm{pH}$ 7.8). ALP activity was measured by mixing the cell extract with $5 \mathrm{mM}$ pNPP (Sigma, St. Louis, MO, USA) in a buffer ( $\mathrm{pH}$ 10.4) containing $0.1 \mathrm{M}$ glycine, $1 \mathrm{mM} \mathrm{MgCl} 2$ and $1 \mathrm{mM}$ $\mathrm{ZnCl}_{2}$ at $37^{\circ} \mathrm{C}$, and measuring the absorbance at 405 $\mathrm{nm}$. In the mineralization analysis, cultured osteoblasts were cultured for 21 days. Treatment with kaempferol $(10 \mu \mathrm{M})$ or $17 \beta$-estradiol $(100 \mathrm{nM})$ in the presence of $\beta$ glycerophosphate $(20 \mathrm{ng} / \mathrm{mL})$ was performed at 3-day intervals. After 21 days of culture, the cells were rinsed with deionized water twice and fixed in $70 \%$ ice-cold 
ethanol for 1 hour at $4^{\circ} \mathrm{C}$. The mineralization assay was performed by staining the cells with $4 \%$ Alizarin Red S (Sigma, St. Louis, MO, USA) for 15 minutes at room temperature and washing them five times with deionized water. The stained cells were then dehydrated with $70 \%$ ethanol followed by absolute ethanol. Cells were observed with phase contrast microscope at a magnification of $20 \times$, and orange-red staining indicated the position and intensity of the calcium deposits. Alizarin red was quantified as previously described [12].

\section{Real-time quantitative PCR}

Total RNA from cultured osteoblasts was isolated by RNAzol ${ }^{\mathbb{R}} \mathrm{RT}$ reagent (Molecular Research Center, Cincinnati, OH, USA), and $5 \mu \mathrm{g}$ of RNA was reverse-transcribed by Moloney murine leukemia virus reverse transcriptase (Invitrogen, CA, USA), according to the manufacturer's instructions. Real-time PCR of COL1A1 (234 bp), osteonectin (182 bp), osteocalcin (281 bp), Runx2 (252 bp), osterix (159 bp) and 18 S rRNA (320 bp) transcripts was performed on equal amounts of reverse-transcribed products, using KAPA $^{\mathrm{TM}} \mathrm{SYBR}^{\circledR}$ FAST qPCR kit according to the manufacturer's instructions (Kapa Biosystems, Cape Town, South Africa). The primers were designed according to the database from genebank (NM_053304 for COL1A1; NM_012656 for osteonectin; NM_013414 for osteocalcin; NM_001146038.1 for Runx2; NM_130458.3 for osterix and NR_003286 for $18 \mathrm{~S}$ rRNA. The SYBR green signal was detected by a Mx3000 $\mathrm{p}^{\mathrm{TM}}$ multiplex quantitative PCR machine (Stratagene, La Jolla, CA, USA). The relative levels of transcript expression were quantified by using the $\Delta \Delta \mathrm{Ct}$ method [17]. The calculation was done by using the $\mathrm{Ct}$ value of $18 \mathrm{~S}$ rRNA to normalize the $\mathrm{Ct}$ value of the target gene in each sample to obtain the $\Delta \mathrm{Ct}$ value, which then was used for comparison among different samples. The PCR products were analyzed by gel electrophoresis, and the specificity of amplification was confirmed by the melting curve.

\section{Protein assay}

Protein concentrations were measured routinely by Bradford's method with a kit from Bio-Rad Laboratories (Hercules, CA, USA).

\section{Statistical analysis}

Independent $t$-test was carried out with SPSS software (version 13.0, SPSS, Chicago, IL, USA). $P$ values were corrected by the Bonferroni method for multiple comparison. The level of statistical significance was $P<0.05$.

\section{Results and discussion}

ERs are the members of the superfamily of ligand-regulated nuclear transcription factors. ER $\alpha$ and ER $\beta$, have been identified in cultured rat osteoblasts [12] and estrogen was shown to stimulate the differentiation of osteoblasts (Additional file 1). Thirty-six flavonoids, mainly derived from vegetables and Chinese herbs, were screened for their ability to actively stimulate osteoblast differentiation [12], and kaempferol (Figure 1A) was one of the positive hits. The estrogenic activity of the kaempferol was determined by its induction effect on pERE-Luc-transfected cultured osteoblasts (Figure 1B, upper panel). $17 \beta$-estradiol was used as the positive control and induced pERE-Luc activity about 3-fold upon treatment $(P=0.041)$. In osteoblasts expressing pERELuc, kaempferol induced luciferase activity in a dosedependent manner: the luciferase activity increased to $3.98 \pm 0.31$ folds (at $50 \mu \mathrm{M}, P=0.038$ ) after treatment as shown in Figure 1B. These activities showed the authenticity of pERE-Luc construct. Kaempferol treatment, even at rather high concentrations, did not affect cell viability; therefore, toxic side effects within the dose range investigated can be excluded (Additional file 2). In addition, pre-treatment with ICI 182,780 fully blocked kaempferol-induced pERE-Luc activity, indicating that kaempferol acts via ER activation. Kaempferol was able to trigger ER $\alpha(\sim 66 \mathrm{kDa})$ phosphorylation at the S118 position in a time-dependent manner in cultured osteoblasts, generating a 7 -folds increase at 30 minutes of treatment (Figures $1 \mathrm{C}$ and 1D), which could serve as further evidence of its estrogenic property. $17 \beta$-estradiol served as a positive control with a nearly 10 -folds increase in ER $\alpha$ phosphorylation at 30 minutes, while ICI 182,780 completely blocked kaempferol-induced $\mathrm{ER} \alpha$ phosphorylation. In all cases, the total amount of ER $\alpha$ remained unchanged.

The effects of kaempferol on osteoblastic differentiation were determined in cultured osteoblasts. Application of kaempferol in the cultures induced ALP activity in a dose-dependent manner (Figure 2A): $2.08 \pm 0.29$ folds induction was reached with about $30 \mu \mathrm{M}$ kaempferol. In comparison with the ALP activity induced by $17 \beta$-estradiol (1.98 \pm 0.19 folds increase), the effect of kaempferol was more robust. Osteoblastic ALP activities induced by both $17 \beta$-estradiol and kaempferol were fully blocked by ICI 182,780 (Figure 2A, $\mathrm{P}=0.0412$ and $P=0.0485$, respectively). Transcription of genes for several bone differentiation markers, (COL1A1, osteonectin, osteocalcin, Runx 2 and osterix, was up-regulated with $17 \beta$-estradiol and kaempferol treatment in cultured osteoblasts. In all cases, the transcripts encoding these markers were markedly induced 3 to 4 -folds by $100 \mathrm{nM} 17 \beta$-estradiol and 2 to 3 -folds by $10 \mu \mathrm{M}$ kaempferol. The inductions were completely blocked by pre-treatment with ICI $182,780$ (Figure $2 \mathrm{~B})$. The statistically significant results include the blocking effects of $17 \beta$-estradiol $(P=0.0012$ for COL1A1; $P=0.0070$ for osteonectin; $P=0.0033$ for 


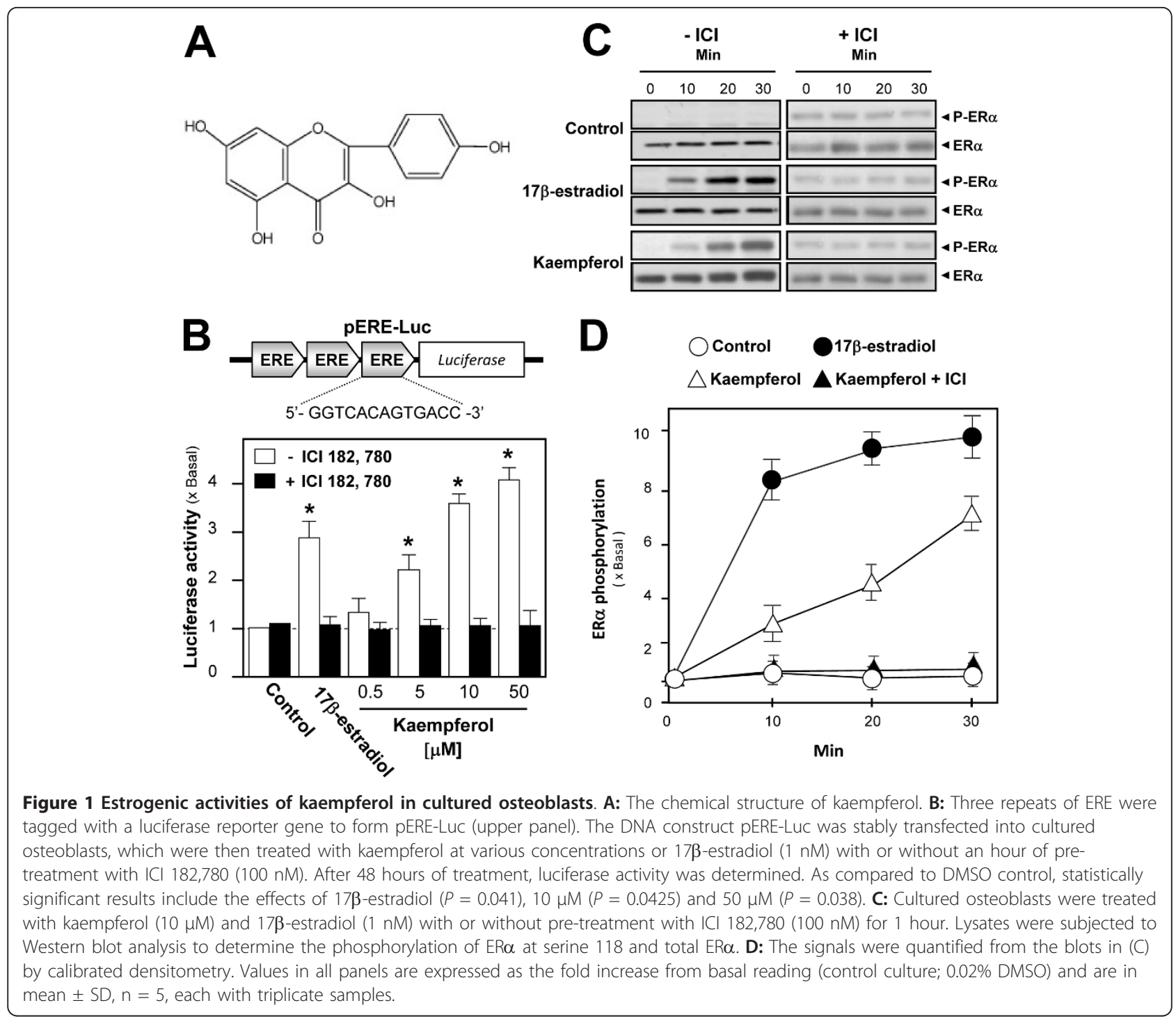

osteocalcin; $P=0.0441$ for osterix and $P=0.0023$ for Runx2) and kaempferol $(P=0.0065$ for COL1A1; $P=$ 0.0063 for osteonectin; $P=0.0072$ for osteocalcin; $P=$ 0.0068 for osterix and $P=0.0064$ for Runx2). In addition, both $17 \beta$-estradiol and kaempferol induced osteoblastic mineralization $(3.27 \pm 0.34$ folds and $4.02 \pm 0.41$ folds, respectively), which was fully blocked by the treatments with ICI 182,780 (Figures 2C and 2D). The kaempferol results were similar to those of the $17 \beta$ estradiol treatment, suggesting that the osteogenic property of kaempferol was entirely due to its estrogenic properties. The statistically significant results include the blocking effects of $17 \beta$-estradiol $(P=0.0093)$ and kaempferol $(P=0.0085)$.

Furthermore, pre-treatment with DKK-1, a Wnt receptor inhibitor, could not block kaempferol-induced effects (Figure 3A). The specific inhibition by ICI
182,780 (Figure 2B) but not by DKK-1 confirmed that kaempferol-induced osteogenic effects were mediated by the activation of ER $\alpha$ via a classical ER signaling pathway. In addition, kaempferol did not show any activation of Wnt/ $\beta$-catenin signaling, as tested by a luciferase reporter pWRE-Luc (Figure 3B). All evidence collectively indicates that kaempferol-induced osteoblast differentiation is mediated by ER signaling.

As an estrogen alternative without the associated adverse effects of the hormone, flavonoids, a large group of naturally occurring compounds with estrogen-like activities and a valuable potential source for new dietary health interventions for post-menopausal women, have been intensively investigated for their ability in preventing postmenopausal bone loss [18]. Flavonoids have long been recognized to possess a wide range of biological activities [19], and the mechanisms for these activities are being 

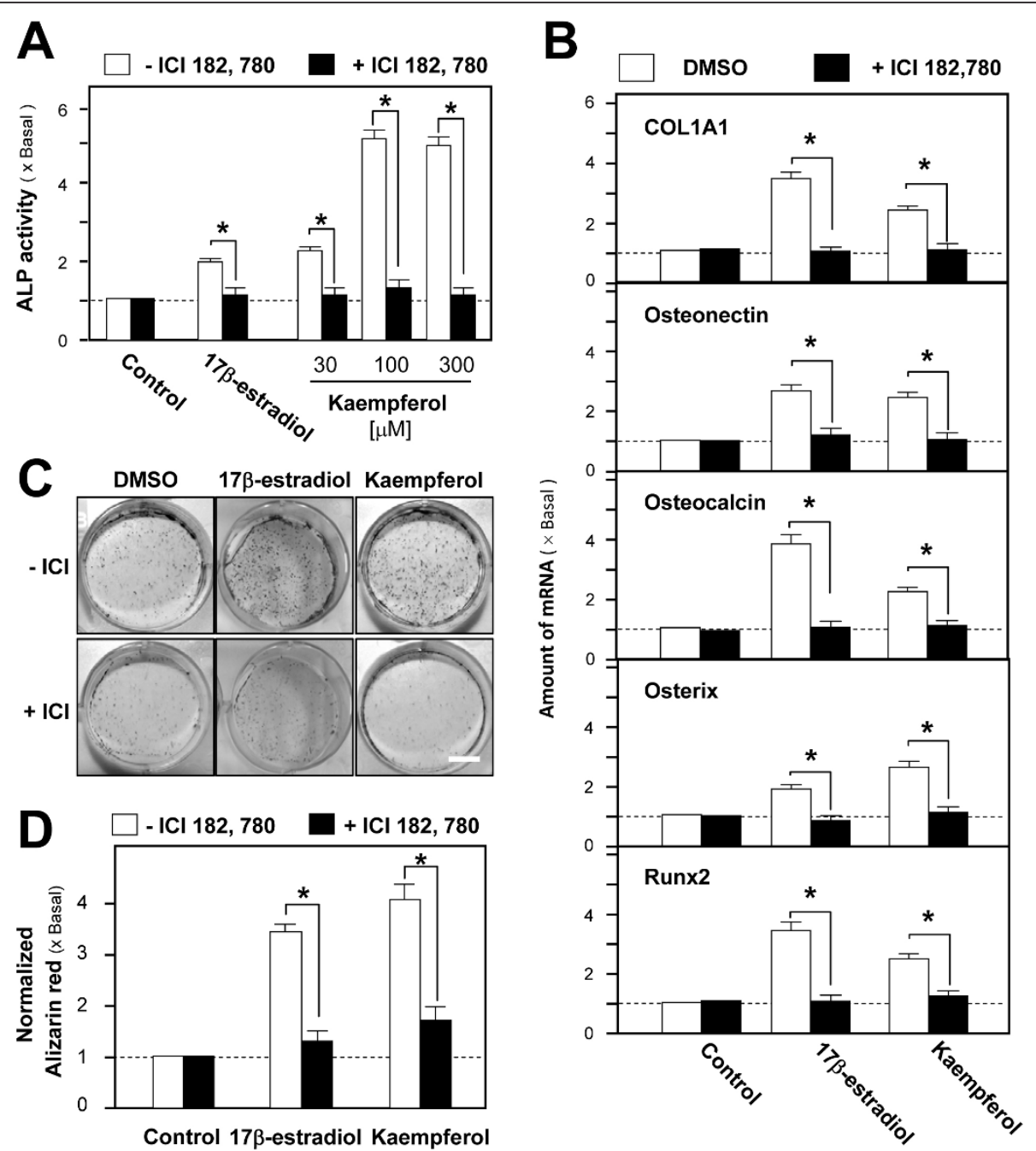

Figure 2 Kaempferol-induced osteogenic differentiation is mediated by ER signaling cultured osteoblasts. A: Application of 17ß-estradiol $(100 \mathrm{nM})$ or kaempferol $(30-300 \mu \mathrm{M})$ in cultured osteoblasts for 3 days increased ALP activity in a dose-dependent manner. The stimulatory effect was abolished upon pre-treatment with ICI 182,780 (100 nM) for 1 hour. The ALP activities detected after the pre-treatment of ICI 182, 780 were compared with the ALP activities detected without the pre-treatment. The statistically significant results include the blocking effects of $17 \beta$ estradiol $(P=0.0412)$, kaempferol at $30 \mu \mathrm{M}(P=0.0485), 100 \mu \mathrm{M}(P=0.0081)$ and $300 \mu \mathrm{M}(P=0.0086)$. B: Cultured osteoblats were treated with $17 \beta$-estradiol $(100 \mathrm{nM})$ or kaempferol $(10 \mu \mathrm{M})$ for 2 days, with or without pre-treatment with $\mathrm{ICI} 182,780(100 \mathrm{nM})$ for 1 hour. Total RNAs were extracted from the cultures to perform quantitative PCR for osteogenesis-associated genes, including type I collagen (COL1A1), osteonectin, osteocalcin, osterix and Runx2 mRNAs. The mRNA amounts of osteogenesis-associated genes detected after the pre-treatment of ICI 182, 780 were compared with the mRNA amounts detected without the pre-treatment. The statistically significant results include the blocking effects of 17 $\beta$-estradiol $(P=0.0012$ for COL1A1; $P=0.0070$ for osteonectin; $P=0.0033$ for osteocalcin; $P=0.0441$ for osterix and $P=0.0023$ for Run 2 ) and kaempferol $(P=0.0065$ for COL1A1; $P=0.0063$ for osteonectin; $P=0.0072$ for osteocalcin; $P=0.0068$ for osterix and $P=0.0064$ for Runx2). C: Cultured osteoblasts underwent mineralization upon the addition of $17 \beta$-estradiol $(100 \mathrm{nM})$ or kaempferol $(10 \mu \mathrm{M})$ in the presence of $\beta$ glycerophosphate $(5 \mathrm{mM})$. After 21 days of treatment, nodules were found, as shown by Alizarin Red staining. The mineralization process was hindered by pre-treatment with ICI 182,780 (100 nM). D: From the cultures of (C), Alizarin Red staining was quantified using a solution of $20 \%$ methanol and 10\% acetic acid in water, and the reading was done on a spectrophotometer at $450 \mathrm{~nm}$. The normalized alizarin red amounts detected after the pre-treatment of ICI 182, 780 were compared with the amount detected without the pre-treatment. The statistically significant results include the blocking effects of $17 \beta$-estradiol $(P=0.0093)$ and kaempferol $(P=0.0085)$. Values in all panels are expressed as the fold increase from the basal reading (control culture; $0.02 \% \mathrm{DMSO}$ ); mean $\pm S D, n=5$, each with triplicate samples.

actively explored. Kaempferol is known to be the most abundant phytoestrogen in Western diets as compared with soybean isoflavones [18]. This compound is widely found in many food plants [11,20].
Previously, we have shown that the flavone baicalin, which is derived from the roots of Scutellaria baicalensis, possesses stimulatory effects on osteoblast differentiation [12]. This baicalin-induced bone effect was not 

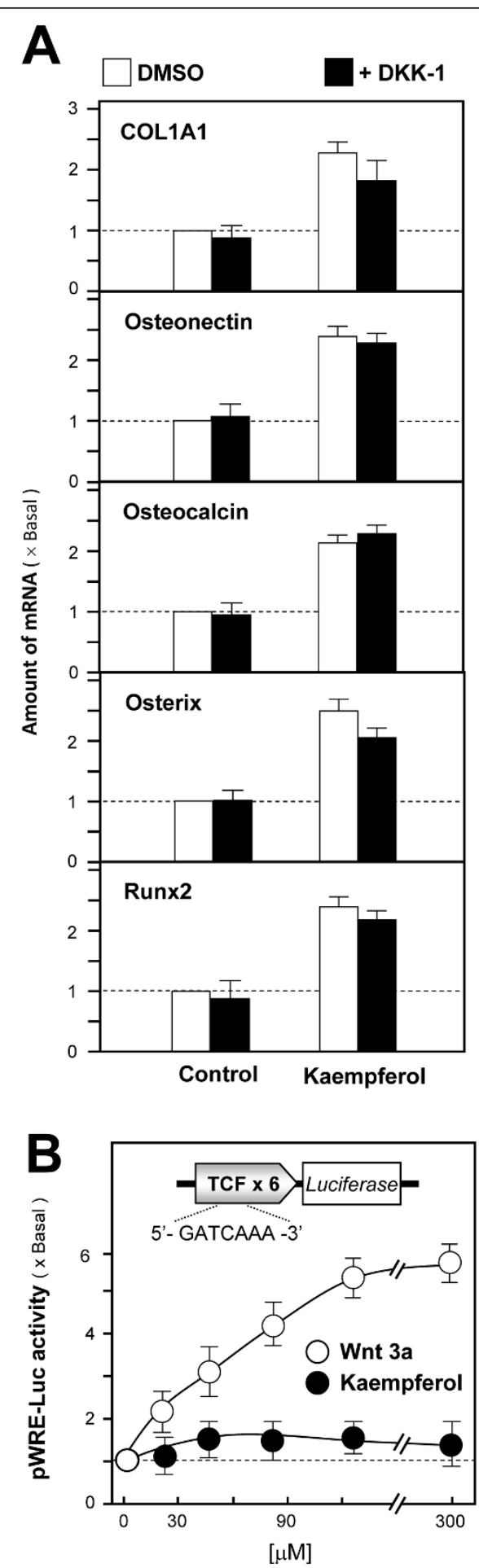

Figure 3 Kaempferol-stimulated osteogenic effect is not mediated by activation of the $W n t / \beta$ catenin pathway. A: Cultured osteoblasts were treated with kaempferol $(10 \mu \mathrm{M})$ for 2 days, with or without DKK-1 pre-treatment $(0.2 \mathrm{mg} / \mathrm{mL})$ for 1 hour. Total mRNA was extracted from the cultures to perform quantitative PCR for osteogenesis-associated genes, including type I collagen
(COLIA1), osteonectin, osteocalcin, Runx2 and osterix. B: A reporter construct corresponding to pWRE-Luc (upper panel) was used as described previously. pWRE-Luc was transfected into cultured osteoblasts for 2 days before addition of Wnt3a (200 ng/mL; the ligand of Wnt/ $\beta$-catenin pathway) or kaempferol (10 $\mu \mathrm{M})$. Fortyeight hours later, luciferase activity was assayed. Values are expressed as the fold increase from the basal reading (control culture; $0.02 \% \mathrm{DMSO}$ ); mean $\pm \mathrm{SD}, \mathrm{n}=5$, each with triplicate samples.

mediated by its estrogenic property. Rather, baicalin promoted osteogenesis via regulation of the $\mathrm{Wnt} / \beta$-catenin signaling cascade. In the present study, kaempferol was shown to enhance osteoblastic differentiation and mineralization via ER signaling by inducing ER $\alpha$ phosphorylation and transcriptional activity of ERE. Unlike baicalin, kaempferol did not activate the $\mathrm{Wnt} / \beta$-catenin pathway, and the kaempferol-induced osteogenic effects could not be abolished upon treatment with the Wnt receptor inhibitor DKK-1. Taken together, these results suggested that the osteogenic effects mediated by flavonoids could be dependent or independent of their estrogenic properties. This idea is supported by our previous screening of different flavonoids, encompassing the major sub-classes, which showed that the estrogenic activities of selective flavonoids are not correlated with osteogenic activities [12]. Moreover, our results also implied that different flavonoids could promote bone differentiation via different signaling mechanisms and these mechanisms of action and signaling cascades should be further studied. Further, combining flavonoids that regulate bone differentiation via different mechanisms could have synergistic or additive effects, which could be further verified. The potential of kaempferol as a bone-promoting flavonoid should be investigated for developing potential drugs or food supplements for the prevention of the bone loss associated with menopause.

\section{Conclusion}

Our results showed that kaempferol stimulates osteogenic differentiation of cultured osteoblasts by acting through the estrogen receptor signaling.

\section{Additional material}

Additional file 1: Figure 1. Treatment of kaempferol did not affect the cell viability of cultured osteoblasts. Cultured osteoblasts were challenge with $7 \beta$-estradiol $(10 \mathrm{nM})$, or different doses of kaempferol for 2 day, and the viability was determined by MTT assay. Values are expressed as the $\%$ of the control reading (control cultured treated with $0.02 \%$ DMSO), and are in mean \pm , where $n=4$, each with triplicate samples.

Additional file 2: Figure 2. Estrogen induces osteogenic effect in cultured rat osteoblasts. The osteogenic expressions of estrogen receptors was determined in cultured rat osteoblasts. 


\section{Abbreviations}

ALP: Alkaline phosphatase; COL1A1: Type I collagen; DMSO: Dimethyl sulfoxide; DKK-1: Dickkopf related protein 1; ER: Estrogen receptor; ERE: Estrogen responsive element; PNPP: P-nitrophenyl-phosphate; Runx2: Runtrelated transcription factor 2

\section{Acknowledgements}

This research was supported by grants from Research Grants Council of Hong Kong (HKUST 6419/06 M, N_HKUST629/07, 662608, 661110) and Croucher Foundation (CAS-CF07/08.SC03) to KWKT and DTWL.

\section{Author details}

'Division of Life Science and Center for Chinese Medicine, State Key Laboratory of Molecular Neuroscience, The Hong Kong University of Science and Technology, Clearwater Bay, New Territories, Hong Kong SAR, China. ${ }^{2}$ Institute of Chinese Materia Medica, Shanghai University of Traditional Chinese Medicine 1200, Cailun Road, Zhangjiang Hi-Tech Park, Shanghai 201203, China. ${ }^{3}$ Molecular Cell Physiology and Endocrinology, Technische Universität Dresden, Institut für Zoologie, Professur für Molekulare Zellphysiologie und Endokrinologie, 01062 Dresden, Germany.

\section{Authors' contributions}

$K T, R C, T D, Z W$, and GV designed the study and wrote the manuscript. AG conducted the experiments and drafted the manuscript. KZ and VC assisted in performing the experiments. All authors read and approved the final version of the manuscript.

\section{Competing interests}

The authors declare that they have no competing interests.

Received: 21 June 2011 Accepted: 30 April 2012

Published: 30 April 2012

\section{References}

1. Riggs BL, Khosla S, Melton LJ: Sex steroids and the construction and conservation of the adult skeleton. Endocr Rev 2002, 23:279-302.

2. Delmas PD: Treatment of post-menopausal osteoporosis. Lancet 2002, 359:2018-2026

3. Chien KR, Karsenty G: Longevity and lineages: toward the integrative biology of degenerative diseases in hearth, muscle, and bone. Cell 2005, 120:533-544.

4. Rodan GA, Martin TJ: Therapeutic approaches to bone diseases. Science 2000, 289:1508-1514

5. Arimandi BH, Alekel L, Hollis BW, Amin D, Stacewicz-Sapuntzakis M, Guo P, Kukreja SC: Dietary soybean protein prevents bone loss in an ovariectomized rat model of osteoporosis. J Nutr 1996, 126:161-167.

6. Hegarty VM, May HM, Khaw KT: Tea drinking and bone mineral density in older women. Am J Clin Nutr 2000, 71:1003-1007.

7. Muhlauer RC, Li F: Effect of vegetables on bone metabolism. Nature 1999, 401:343-344.

8. Huang L, Yagura T, Chen S: Sedative activity of hexane extract of Keampferia galanga L. and its active compounds. J Ethnopharmacol 2008, 120:123-125.

9. Wattel A, Kamel S, Mentaverri R, Lorget F, Prouillet C, Petit JP, Fardelonne P, Brazier M: Potent inhibitory effect of naturally occurring flavonoids quercetin and kaempferol on in vitro osteoclastic bone resorption. Biochem Pharmacol 2003, 65:35-42.

10. Miyake M, Arai N, Ushio S, Iwaki K, Ikeda M, Kurimoto M: Promoting effect of kaempferol on the differentiation and mineralization of murine preosteoblastic cell line MC3T3-E1. Biosci Biotechnol Biochem 2003, 67:1199-1205

11. Prouillet C, Maziere JC, Maziere C, Wattel A, Brazier M, Kamel S: Stimulatory effect of naturally occurring flavonols quercetin and kaempferol on alkaline phosphatase activity in MG-63 human osteoblasts through ERK and estrogen receptor pathway. Biochem Pharmacol 2004, 67:1307-1313.

12. Guo AJ, Choi RC, Cheung AW, Chen VP, Xu SL, Dong TT, Chen JJ, Tsim KW: Baicalin, a flavone, induces the differentiation of cultured osteoblasts: an action via the wnt/ $\beta$-catenin signaling. J Biol Chem 2011, 286.27882-27893.
13. Orriss IR, Knight GE, Ranasinghe S, Burnstock G, Arnett TR: Osteoblast responses to nucleotides increase during differentiation. Bone 2006 39:300-309.

14. Choi RC, Gao QT, Cheung AW, Zhu JT, Lau FT, Li J, Li WZ, Chu GK, Duan R, Cheung JK, Ding AW, Zhao KJ, Dong TT, Tsim KW: A Chinese herbal decoction, Danggui Buxue Tang, stimulates proliferation, differentiation and gene expression of cultured osteosarcoma cells: genomic approach to reveal specific gene activation. Evid Based Complement Alternat Med 2009 .

15. Zhu JT, Choi RC, Chu GK, Cheung AW, Gao QT, Li J, Jiang ZY, Dong TT, Tsim KW: Flavonoids possess neuroprotective effects on cultured pheochromocytoma PC12 cells: a comparison of different flavonoids in activating estrogenic effect and in preventing beta-amyloid-induced cell death. J Agric Food Chem 2007, 55:2438-2445.

16. Zheng KY, Choi RC, Xie HQ, Cheung AW, Duan R, Guo AJ, Zhu JT, Chen VP, Bi CW, Zhu KY, Lau DT, Dong TT, Lau BW, Tsim KW: Ligustilide suppresses the biological properties of Danggui Buxue Tang: a Chinese herbal decoction composed of Radix Astragali and Radix Angelica Sinensis. Planta Med 2010, 76:439-443.

17. Winer J, Jung CK, Shackel I, Williams PM: Development and validation of real-time quantitative reverse transcriptase-polymerase chain reaction for monitoring gene expression in cardiac myocytes in vitro. Anal Biochem 1999, 270:41-49.

18. Coxam V: Phyto-estrogens and bone health. Proc Nutr Soc 2008 67:184-195.

19. Middleton E, Kandaswami C, Theoharides TC: The effects of plant flavonoids on mammalian cells: implications for inflammation, heart disease, and cancer. Pharmacol Rev 2000, 52:673-751.

20. Pang JL, Ricupero DA, Huang S, Fatma N, Singh DP, Romero JR, Chattopadhyay N: Differential activity of kaempferol and quercetin in attenuating tumor necrosis factor receptor family signaling in bone cells. Biochem Pharmacol 2006, 71:818-826.

doi:10.1186/1749-8546-7-10

Cite this article as: Guo et al:: Kaempferol as a flavonoid induces osteoblastic differentiation via estrogen receptor signaling. Chinese Medicine 2012 7:10.

\section{Submit your next manuscript to BioMed Central and take full advantage of:}

- Convenient online submission

- Thorough peer review

- No space constraints or color figure charges

- Immediate publication on acceptance

- Inclusion in PubMed, CAS, Scopus and Google Scholar

- Research which is freely available for redistribution

Submit your manuscript at www.biomedcentral.com/submit
C) Biomed Central 